\title{
Variations of Tongue Coating Microbiota in Patients with Gastric Cancer
}

\author{
Jie Hu, ${ }^{1}$ Shuwen Han, ${ }^{1}$ Yan Chen, ${ }^{1}$ and Zhaoning $\mathrm{Ji}^{2}$ \\ ${ }^{1}$ Department of Oncology, Wannan Medical College, Wuhu, Anhui 241000, China \\ ${ }^{2}$ The Cancer Center, Yijishan Hospital of Wannan Medical College, Wuhu, Anhui 241000, China
}

Correspondence should be addressed to Zhaoning Ji; jizn1101@163.com

Received 16 June 2015; Revised 27 August 2015; Accepted 3 September 2015

Academic Editor: Janusz Blasiak

Copyright (C) 2015 Jie Hu et al. This is an open access article distributed under the Creative Commons Attribution License, which permits unrestricted use, distribution, and reproduction in any medium, provided the original work is properly cited.

\begin{abstract}
The physical status of humans can be estimated by observing the appearance of the tongue coating, known as tongue diagnosis. The goals of this study were to reveal the relationship between tongue coating appearance and the oral microbiota in patients with gastric cancer and to open a novel research direction supporting tongue diagnosis. We used a tongue manifestation acquisition instrument to analyse the thickness of the tongue coating of patients with gastric cancer and that of healthy controls, and highthroughput sequencing was used to describe the microbial community of the tongue coating by sequencing the V2-V4 region of the $16 \mathrm{~S}$ rDNA. The tongue coatings of 74 patients with gastric cancer were significantly thicker than those of 72 healthy controls $(343.11 \pm 198.22$ versus $98.42 \pm 48.25, P<0.001) ; 51.35 \%$ of the patients were assessed as having thick tongue coatings, whereas all healthy controls were assessed as having thin tongue coatings. Thick tongue coatings presented lower microbial community diversity than thin tongue coatings. The tongue coating bacterial community is associated with the appearance of the tongue coating. The tongue coating may be a potential source for diagnosing gastric cancer, but its sensitivity needs to be further improved.
\end{abstract}

\section{Introduction}

Traditional Chinese Medicine (TCM), practiced by generations of Chinese doctors for over 3000 years [1, 2], has been abundantly used in the clinic. As an essential part of TCM, tongue diagnosis estimates human physical status by observing the appearance of the tongue coating. The normal tongue is covered by a thin, white coating that is composed of bacteria, desquamated epithelial cells, leukocytes, blood metabolites, and various nutrients. When the human body is in a morbid state, with less food and chewing, the tongue coating is thicker. Tongue diagnosis has many advantages as it is noninvasive and convenient. However, it is not widely approved due to its subjectivity and nonreproducibility because it is primarily estimated by the naked eye by experienced TCM doctors. To eliminate the influence of artificial factors, a computer-aided tongue diagnosis system has been developed to establish a uniform standard for tongue diagnosis $[3,4]$.

A variety of microbiota exist throughout the human body and play critical roles in the formation of various human diseases. Many studies have associated microbes in the human body with the initiation and progression of particular diseases, such as obesity [5], coronary heart disease $[6,7]$, and colorectal cancer [8-10]. Recently, a growing number of studies have investigated the relationship between the oral microbial community and diseases. Some studies have demonstrated that patients with pancreatic cancer [11] and patients with inflammatory bowel disease [12] have unique oral microbial community structures, and the oral microbial community may be a potential biomarker source.

Gastric cancer, as one of the most common malignancies causing death, has been considered to be the result of environmental and genetic factors. However, the specific mechanism remains obscure. It is widely accepted that Helicobacter pylori, which is commonly found in oral cavity, is a strong inducer of gastric cancer and precancerous lesions [13-15]. Epidemiological studies have also implicated human oral bacteria in the aetiology of gastrointestinal cancers. Much evidence has implicated oral microbiota. Previously, technological limitations prevented the description of complicated oral 
microbes. The development of next-generation sequencing technology makes it possible to comprehensively describe the microbiota in the oral cavity.

In the present study, a tongue manifestation acquisition instrument was used to compare the thickness of the tongue coating of patients with gastric cancer and that of healthy controls. In addition, next-generation sequencing was used to describe the tongue coating microbiota of patients with different thicknesses of tongue coatings and healthy controls.

\section{Materials and Methods}

2.1. Individual Screening. Seventy-four patients with histologically confirmed gastric cancer (GC) in Yijishan Hospital (Wannan Medical College First Affiliated Hospital) from November 2013 to April 2014 and 72 volunteers who had no stomach discomfort over the past three years were recruited as cases and healthy controls (HCs), respectively. The HCs had no malignant tumours, oral diseases, or gastric diseases, and the absence of oral disease among patients with GC was confirmed. To remove chemotherapeutics and surgery as confounding variables for tongue coating microbiota, all patients selected had been diagnosed for 1 day to 26 days and had not undergone any chemotherapy or surgery. None of the subjects had used any antibiotics within the past two months. The use of these subjects was approved by the Yijishan Hospital. All patients and healthy controls in the study provided their informed consent.

2.2. Tongue Image Analysis. All tongue images were photographed in the morning prior to patient food consumption to avoid the interference of food debris, and the thickness of the tongue coatings was analysed by the DS01-B tongue manifestation acquisition instrument (Daosh Co., Shanghai, China). The DS01-B tongue manifestation acquisition instrument consists of a photographic system and a software analysis system that can quantify the thickness of tongue coatings automatically. This instrument has been applied and proven effective in a previous research study [16].

2.3. Sample Collection. All subjects were required to rinse their mouths with saline buffer before sample collection. The tongue coating samples were collected by scraping the tongue surface with 3 sterile swabs 3 times. Then, the sterile swabs were soaked in tubes with $1 \mathrm{~mL}$ of phosphate-buffered saline to wash off the tongue coating adsorbed on the sterile swabs. The tubes were centrifuged for $5 \mathrm{~min}$ at $5000 \mathrm{rpm}$, and the precipitates were collected. Samples were frozen in liquid nitrogen and immediately stored at $-80^{\circ} \mathrm{C}$.

2.4. Gene Amplification. DNA was extracted using Tiangen extraction kits (Tiangen, Peking, China) according to the manufacturer's protocols. The integrity of the genomic DNA was assessed by electrophoresis (1\% agarose gel). We amplified the V2-V4 region (338F-806R) of the 16S rRNA using universal primers [17]. All samples were amplified on an ABI GeneAmp 9700 (ABI, USA) using the following parameters: $94^{\circ} \mathrm{C}$ for $10 \mathrm{~min}$, then 30 cycles of $94^{\circ} \mathrm{C}$ for $30 \mathrm{sec}, 55^{\circ} \mathrm{C}$ for
$30 \mathrm{sec}$, and $72^{\circ} \mathrm{C}$ for $60 \mathrm{sec}$, with a final incubation at $72^{\circ} \mathrm{C}$ for $10 \mathrm{~min}$. PCR reactions were performed in triplicate in a $20 \mu \mathrm{L}$ mixture containing $4 \mu \mathrm{L}$ of $5 \times$ FastPfu Buffer, $2 \mu \mathrm{L}$ of $2.5 \mathrm{mM}$ dNTPs, $0.8 \mu \mathrm{L}$ of each primer $(5 \mu \mathrm{M}), 0.4 \mu \mathrm{L}$ of FastPfu Polymerase, and 10 ng of template DNA.

2.5. Illumina Sequencing. Amplicons were extracted from $2 \%$ agarose gels and purified using the AxyPrep DNA Gel Extraction Kit (Axygen Biosciences, Union City, CA, USA) according to the manufacturer's instructions and quantified using QuantiFluor-ST (Promega, USA). Purified amplicons were pooled in equimolar amounts and paired-end sequenced $(2 \times 300)$ on an Illumina MiSeq platform according to the standard protocols.

2.6. Sequence Analysis. To obtain accurate data, we eliminated reads with more than 2 mismatched bases in the forward primer, cut off low-quality bases from the $3^{\prime}$ end, and discarded reads with ambiguous bases, short reads, or reads with an average accuracy less than 0.2 . Filtered sequences were binned into OTUs with the QIIME pipeline. The OTU is an artificial classification unit utilized in phylogenetics and population genetics studies. OTU clustering is conducted to estimate the number of species or genera in each sample by applying a similarity threshold ( $97 \%$ in our study). To ensure accuracy, a UPARSE pipeline was used to cluster sequences into OTUs [18], and UCHIME was used to eliminate chimaeras among sequences [19]. To perform the taxonomic analysis, each OTU was aligned to the SILVA database [20], and the closest species or genera with confidence levels higher than $70 \%$ were identified. We analysed population structure and richness based on each of the following five categories: phylum, class, order, family, and genus.

2.7. Statistical Analysis. An independent $t$-test was used to calculate relative abundance. Frequency table data were analysed using $\chi^{2}$ analysis or Fisher's exact test. Differences between the groups were evaluated using the StudentNewman-Keuls (SNK) test. All statistical tests were twosided, and a $P$ value less than 0.05 was considered statistically significant.

\section{Results}

3.1. General Characteristics and Tongue Coating Thickness. We used the DS01-B tongue manifestation acquisition instrument to analyse the thickness of tongue coatings and found that the thickness differed between the patients and the healthy controls. To control for factors that may influence the microbiota of tongue coatings, we included BMI [21], diabetes [22], and hypertension [6], which may influence the human body microbial community, and smoking [23] and drinking [24], which may be associated with the risk of gastric cancer, in the statistical analysis. As shown in Table 1, HCs did not significantly differ from GC patients with respect to age, gender, BMI, smoking, drinking, diabetes, or hypertension $(P>0.05)$; however, the thickness of the tongue coating was 
TABLE 1: General characteristics and tongue coating thickness of all subjects.

\begin{tabular}{|c|c|c|c|}
\hline & $\begin{array}{l}\text { Normal } \\
\text { controls } \\
(n=72)\end{array}$ & $\begin{array}{c}\text { Gastric cancer } \\
\text { patients } \\
(n=74)\end{array}$ & $P^{\S}$ \\
\hline Male, $n$ & 35 & 37 & 0.834 \\
\hline $\begin{array}{l}\text { Age }(\text { mean } \pm S D) \text {, } \\
\text { years }\end{array}$ & $54.55 \pm 9.63$ & $57.46 \pm 8.43$ & 0.464 \\
\hline $\begin{array}{l}\mathrm{BMI}(\text { mean } \pm \mathrm{SD}) \\
\mathrm{kg} / \mathrm{m}^{2}\end{array}$ & $22.16 \pm 2.14$ & $23.13 \pm 3.14$ & 0.530 \\
\hline Habit of smoking, $n$ & 12 & 15 & 0.848 \\
\hline Habit of drinking, $n$ & 15 & 16 & 0.902 \\
\hline Diabetes, $n$ & 1 & 2 & 0.959 \\
\hline Hypertension, $n$ & 5 & 5 & 0.850 \\
\hline $\begin{array}{l}\text { Thickness of the } \\
\text { tongue coating }\end{array}$ & $98.42 \pm 48.25$ & $343.11 \pm 198.22$ & $<0.001$ \\
\hline
\end{tabular}

TABLE 2: The number of controls and patients with thin and thick tongue coatings.

\begin{tabular}{lccc}
\hline & $\begin{array}{c}\text { Thin tongue } \\
\text { coating }(\%)\end{array}$ & $\begin{array}{c}\text { Thick tongue } \\
\text { coating }(\%)\end{array}$ & Total \\
\hline $\begin{array}{l}\text { Normal controls } \\
\text { Patients with gastric } \\
\text { cancer }\end{array}$ & $72(100)$ & $0(0)$ & 72 \\
Total & $10(48.65)$ & $38(51.35)$ & 74 \\
\hline
\end{tabular}

significantly different between the two groups $(P<0.001)$. According to the manual of the DS01-B tongue manifestation acquisition instrument, tongue coatings were divided into thick tongue coatings and thin tongue coatings at the threshold of 300 . Tongue coatings valued above 300 were regarded as thick tongue coatings, and coatings with values below 300 were considered thin tongue coatings. Figure 1 is an image analysed by the tongue manifestation acquisition instrument. Using the tongue manifestation acquisition instrument, the tongue coatings of all HCs (value range: $28.8-218.7$ ) and those of 36 of the 74 patients (48.6-288.3) were determined to be thin, whereas the coatings of 38 of the 74 patients (310.1732.2) were considered thick (Table 2). Though all healthy controls had thin tongue coatings, $48.65 \%$ of patients also had thin tongue coatings, indicating that nearly half of the GC patients could not be detected by the tongue manifestation acquisition instrument. Therefore, improving the sensitivity and reliability of this tool is a vital challenge for the future.

\subsection{Diversity of the Microbial Community in Different Thick-} nesses of Tongue Coatings. To obtain better samples for the next-generation sequencing, 40 of 74 patients and 56 of 72 healthy controls were eliminated because of nonstandard operation, resulting in 34 patients and 17 healthy controls as the final research subjects. The tongue coatings of all HCs (value range: $37.2-190.4$ ) and 16 of 34 patients (54.2-273.3) were determined to be thin, whereas the coatings of 18 of 34 patients (310.1-699.2) were considered thick. Therefore, there were the three following groups: HCs with thin tongue coatings $(n=16)$, patients with thin tongue coatings $(n=16)$, and patients with thick tongue coatings $(n=18)$.

To estimate the diversity of the microbial community in the tongue coatings of the three groups, abundance-based coverage estimator (ACE), Chao, and Shannon indices were used to describe the alpha diversity. We compared the alpha diversities of these three groups and found that the number of OTUs in the thick tongue coating group was significantly lower than those of the thin tongue coating group and the control group. The ACE, Chao, and Shannon values showed similar results (Table 3 ). These findings indicate that patients with thick tongue coatings have lower microbial community diversity than patients and healthy people with thin tongue coatings.

3.3. Relative Abundances of Microbes in the Three Groups. An image of the relative abundances of bacteria at the genus level in each of the three groups was produced (Figure 2). The five genera with the greatest relative abundances in patients with thin tongue coatings were Prevotella, Veillonella, Leptotrichia, Lactococcus, and Streptococcus, and those of patients with thick tongue coatings were Prevotella, Streptococcus, Actinomyces, Veillonella, and Leptotrichia. Patients with thick tongue coatings had higher relative abundances of Actinomyces and Streptococcus than the other two groups.

3.4. Species on the Tongue Coating. We also investigated the microbial diversity at the species level. The majority of species were shared among the three groups. A Venn diagram of the diversity at the species level showed that 225 species were shared among the three groups, 32 species were not detected in patients, 47 species were not detected in healthy controls, and 17 species were detected only in thick tongue coatings (Figure 3). However, the diagram suggests that each individual had his or her own characteristic species profile.

\subsection{The Tongue Coating Microbial Community of Patients and} Controls. We compared the tongue coating microbial community of patients and controls. Both patients and controls contained six dominant phyla, Firmicutes, Proteobacteria, Bacteroidetes, Actinobacteria, Fusobacteria, and TM7, which accounted for $99 \%$ of the tongue coating microbes. The relative abundances of Firmicutes, Bacteroidetes, Fusobacteria, and TM7 were similar between patients and controls. However, there were significant differences in the abundances of Proteobacteria (10.85\% versus $28.55 \%$ relative abundance for patients and controls, resp., $P<0.001)$ and Actinobacteria (12.32\% versus $4.46 \%$, resp., $P<0.001$ ), as shown in Figure 4. Proteobacteria accounted for $28.55 \%$ of the tongue coating microbes from GC patients (ranging from $11.70 \%$ to $42.99 \%$ ) and $10.85 \%$ of the tongue coating microbes from $\mathrm{HCs}$ (ranging from $0.11 \%$ to $34.77 \%$ ). Relative abundance levels of less than $15 \%$ were determined for Proteobacteria in only 1 of 16 GC patients but in 25 of 34 HCs. 

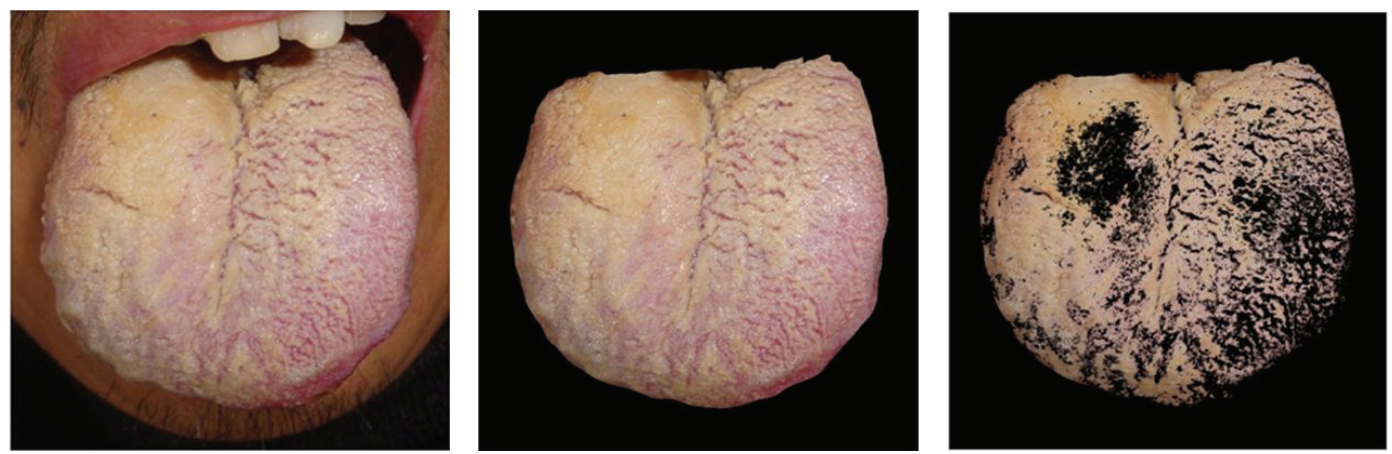

(a) Patient with thick tongue coating
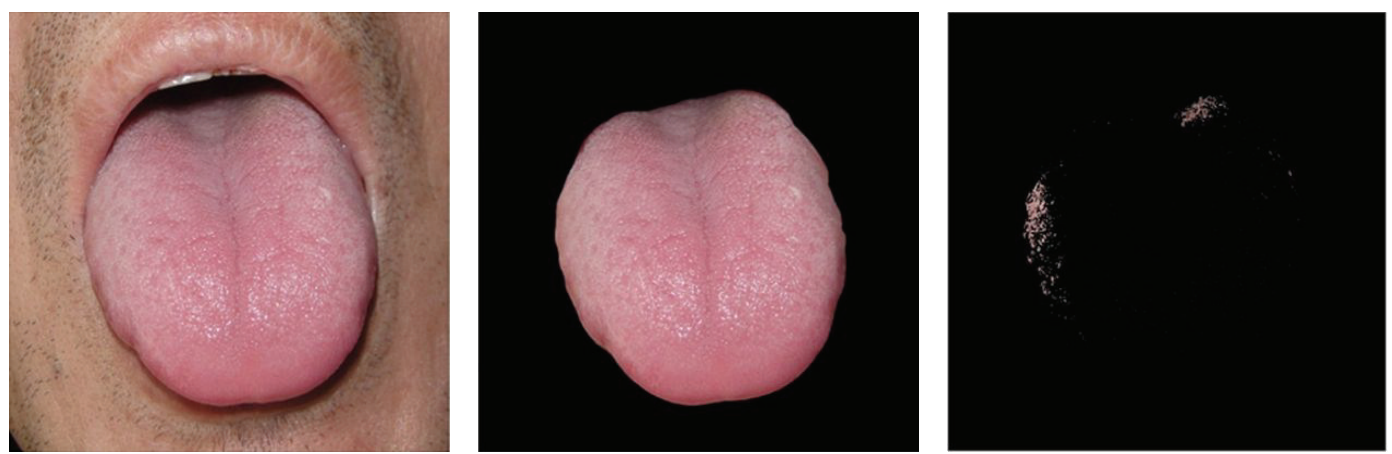

(b) Patient with thin tongue coating
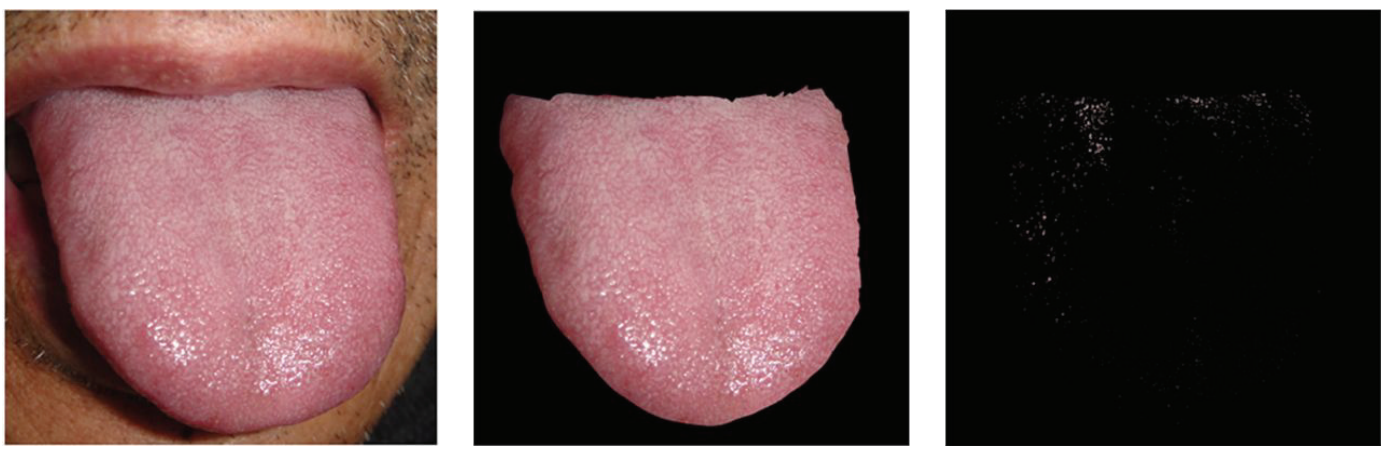

(c) Healthy control with thin tongue coating

FIGURE 1: Images of tongue coatings analysed by a tongue manifestation acquisition instrument. (a) A patient with a typical thick tongue coating, (b) a patient with a thin tongue coating, and (c) a healthy control with a thin tongue coating are shown.

TABLE 3: Tongue coating thickness and the alpha diversity of three groups.

\begin{tabular}{lcccc}
\hline & OTU $($ mean $\pm \mathrm{SD}), n$ & ACE $($ mean $\pm \mathrm{SD})$ & Chao $(\mathrm{mean} \pm \mathrm{SD})$ & Shannon $(\mathrm{mean} \pm \mathrm{SD})$ \\
\hline Normal controls & $153.31 \pm 30.36$ & $205.39 \pm 37.88$ & $198.05 \pm 34.97$ & $3.06 \pm 0.26$ \\
Thin tongue coating group & $151.69 \pm 37.68^{*}$ & $197.54 \pm 11.77^{*}$ & $187.68 \pm 11.13^{*}$ & $3.16 \pm 0.10^{*}$ \\
Thick tongue coating group & $116.06 \pm 36.83^{\#}$ & $160.23 \pm 14.73^{\#}$ & $151.21 \pm 12.48^{\#}$ & $2.85 \pm 0.10^{\#}$ \\
$P^{\S}$ & 0.001 & 0.019 & 0.003 & 0.05 \\
\hline
\end{tabular}

${ }^{\S} P$ values were based on ANOVA.

${ }^{*} P>0.05$ compared with the control group, ${ }^{\#} P<0.05$ compared with the control group and the thin tongue coating group (based on SNK).

At the genus level, 162 genera were identified; the five genera with the highest relative abundances in patients were Prevotella, Streptococcus, Veillonella, Actinomyces, and Leptotrichia, whereas those in controls were Prevotella, Neisseria, Streptococcus, Haemophilus, and Fusobacterium. Compared with the controls, patients had lower relative abundance levels of Fusobacterium (1.78\% versus $6.43 \%, P=0.004)$, Neisseria (4.67\% versus $10.97 \%, P=0.008)$, Haemophilus (1.36\% versus $7.46 \%, P=0.007)$, and Porphyromonas $(0.34 \%$ versus $3.43 \%$, $P=0.002$ ) (Table 4). 
TABLE 4: Relative abundances of selected tongue coating microbial taxa in 34 gastric cancer subjects and 16 control subjects.

\begin{tabular}{|c|c|c|c|}
\hline \multirow{2}{*}{ Taxa (phylum, class, order, family, and genus) } & \multicolumn{3}{|c|}{ Abundance \% } \\
\hline & Case & Control & $P^{\S}$ \\
\hline Proteobacteria (phylum) & 10.85 & 28.55 & $<0.001$ \\
\hline $\begin{array}{l}\text { Proteobacteria, Betaproteobacteria, Neisseriales, Neisseriaceae, and } \\
\text { Neisseria (genus) }\end{array}$ & 4.67 & 10.97 & 0.008 \\
\hline $\begin{array}{l}\text { Proteobacteria, Gammaproteobacteria, Pasteurellales, } \\
\text { Pasteurellaceae, and Haemophilus (genus) }\end{array}$ & 1.36 & 7.46 & 0.007 \\
\hline Actinobacteria (phylum) & 12.32 & 4.46 & $<0.001$ \\
\hline $\begin{array}{l}\text { Fusobacteria, Fusobacteria, Fusobacteriales, Fusobacteriaceae, and } \\
\text { Fusobacterium (genus) }\end{array}$ & 1.78 & 6.43 & 0.004 \\
\hline $\begin{array}{l}\text { Bacteroidetes, Bacteroidia, Bacteroidales, Porphyromonadaceae, and } \\
\text { Porphyromonas (genus) }\end{array}$ & 0.34 & 3.43 & 0.002 \\
\hline
\end{tabular}

${ }^{\S} P$ values were based on $t$-tests (two-sided).

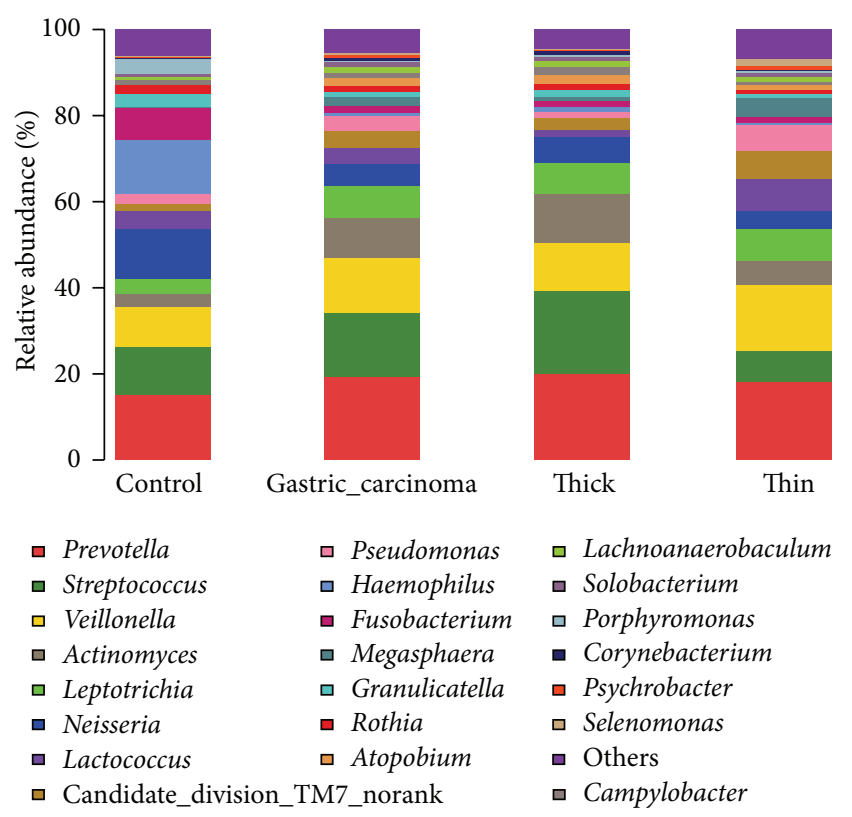

FIGURE 2: Relative abundance at the genera level. The five most abundant genera found in patients with thin tongue coatings were Prevotella, Veillonella, Leptotrichia, Lactococcus, and Streptococcus, and the five most abundant genera in patients with thick tongue coatings were Prevotella, Streptococcus, Actinomyces, Veillonella, and Leptotrichia.

\section{Discussion}

This study is among the first to systematically profile the microbiota in tongue coating samples of patients with GC. In the present study, we found that the tongue coatings of patients with GC were significantly thicker than those of $\mathrm{HCs}$, the tongue coating microbiota community was correlated with the appearance of the tongue coating, and patients with thick tongue coatings had decreased microbial community diversity compared with those of patients and healthy people with thin tongue coatings. These observations may promote tongue coating as a potential diagnostic resource for gastric

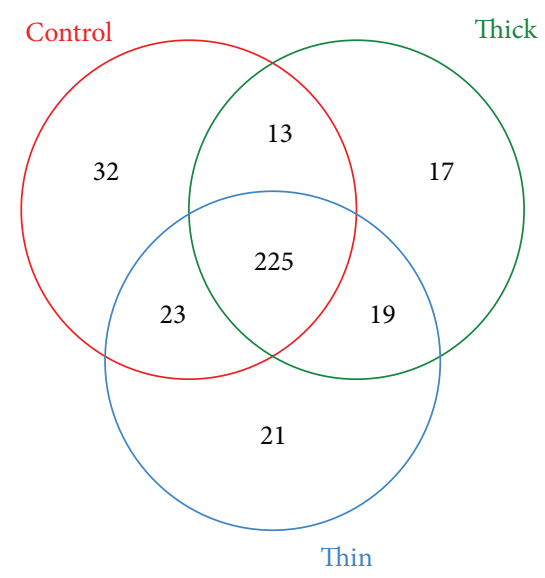

Unique objects: all $=350 ; \mathrm{S} 1=293 ; \mathrm{S} 2=285 ; \mathrm{S} 3=274$

FIgURE 3: A Venn diagram showing the species-level diversity of the three groups. Two hundred and twenty-five species were shared by three groups, 32 species were not detected in patients, 47 species were not detected in healthy controls, and 17 species were detected only in thick tongue coatings.

cancer, which should be sufficiently noninvasive and inexpensive to allow widespread applicability. Compared with other invasive examinations, tongue coating examinations are easier to conduct and may be better accepted by patients.

Compared with previous studies [25-27], we obtained consistent results for the following six dominant phyla from our samples: Firmicutes, Proteobacteria, Bacteroidetes, Actinobacteria, Fusobacteria, and TM7. Our data showed significant differences in the tongue coating microbiota composition between GC patients and HCs. Analysis at the phyla level revealed that the relative abundance of Proteobacteria was significantly lower in GC patients than in $\mathrm{HCs}(P<$ 0.001), which was mainly attributed to lower abundances of Neisseria and Haemophilus. This finding is consistent with results from a previous study [12]. Fusobacterium and Porphyromonas, which contribute to periodontal disease [28, 29], were also less abundant in GC patients than in HCs. 

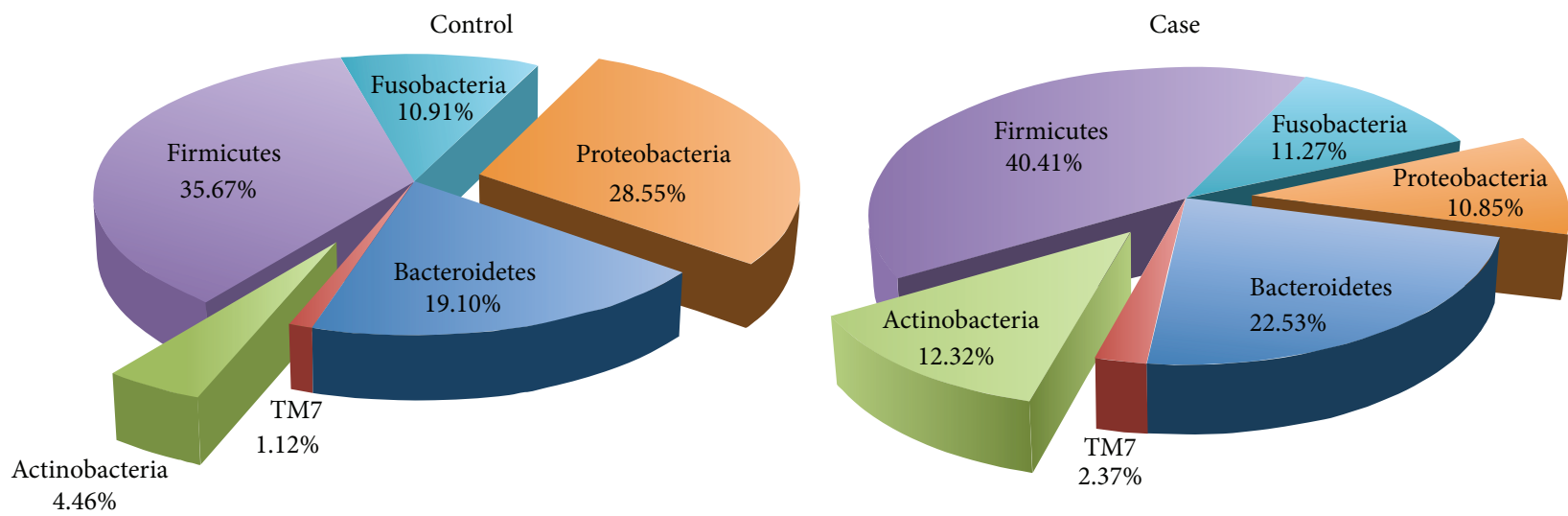

Figure 4: Relative abundances of dominant phyla in cases and controls. The dominant phyla were Firmicutes, Proteobacteria, Bacteroidetes, Actinobacteria, Fusobacteria, and TM7. The abundance of Proteobacteria was $28.55 \%$ in controls compared with $10.85 \%$ in cases, whereas the abundance of Actinobacteria was $4.46 \%$ in controls compared with $12.32 \%$ in cases.

Their prevalence was reported to have an impact on the risk of colorectal cancer [30]. Our study describing the tongue coating microbial community of patients with GC may lead to the development of tongue coating analysis as a microberelated tool for the early diagnosis of gastric cancer.

The relationship between oral microbiota and gastric cancer should be deeply investigated. Some former studies have indicated that some factors, such as poor oral hygiene [31], tooth loss [32, 33], and the metabolism of oral microbes [34], may influence the risk of gastric cancer [35]. In future studies, we will further investigate the interplay of oral microbiota and gastric microbiota, which may become a biological approach for gastric cancer prevention.

In our study, a computer-aided tongue diagnosis system was used to measure the thickness of tongue coatings. Although the system has many advantages, its reliability is questionable. Nearly half of GC patients could not be detected by the system; therefore, the system can serve as only an assisted screening method in clinical work. It cannot serve as an independent and standard diagnostic method unless its sensitivity is greatly improved.

Our study also has some limitations. First, the limited sample size may have caused bias due to human sample variation, sample preparation techniques, and other existing medical conditions. Second, approximately half of the patients with GC in our study possessed thin tongue coatings similar to those of healthy people; therefore, improving the accuracy of tongue coating evaluation to serve as an early diagnosis tool of GC is a serious challenge. Further investigations should use higher sample sizes and aim at revealing how microbes participate in diseases. Regardless, as there is a lack of breakthroughs in cancer screening, analysis of tongue coating microbiota could be an innovative source for gastric cancer screening and diagnosis.

\section{Conflict of Interests}

The authors declare no conflict of interests.

\section{Acknowledgments}

The authors thank the patients and volunteers for allowing them to collect samples. They thank Major Biological Co., Ltd., for their technological support. This study was supported by the National Natural Science Foundation of China (81241102) and the Traditional Chinese Medicine Study of the Anhui Provincial Commission of Health and Family Planning (2012ZY59).

\section{References}

[1] J. Wu, Y. Zhang, and J. Bai, "Tongue area extraction in tongue diagnosis of traditional Chinese medicine," in Proceedings of the IEEE 27th Annual International Conference of the Engineering in Medicine and Biology Society (EMBS '05), pp. 4955-4957, Shanghai, China, January 2005.

[2] G. Nestler, "Traditional Chinese medicine," Medical Clinics of North America, vol. 86, no. 1, pp. 63-73, 2002.

[3] B. Pang, D. Zhang, N. Li, and K. Wang, "Computerized tongue diagnosis based on Bayesian networks," IEEE Transactions on Biomedical Engineering, vol. 51, no. 10, pp. 1803-1810, 2004.

[4] H. Z. Zhang, K. Q. Wang, D. Zhang, B. Pang, and B. Huang, "Computer aided tongue diagnosis system," in Proceedings of the 27th Annual International Conference of the Engineering in Medicine and Biology Society (IEEE-EMBS '05), pp. 6754-6757, IEEE, Shanghai, China, September 2005.

[5] J. Chen, X. He, and J. Huang, "Diet effects in gut microbiome and obesity," Journal of Food Science, vol. 79, no. 4, pp. R442R451, 2014.

[6] G. K. Hansson, "Inflammation, atherosclerosis, and coronary artery disease," The New England Journal of Medicine, vol. 352, no. 16, pp. 1626-1695, 2005.

[7] Z. Wang, E. Klipfell, B. J. Bennett et al., "Gut flora metabolism of phosphatidylcholine promotes cardiovascular disease," Nature, vol. 472, no. 7341, pp. 57-65, 2011.

[8] T. Wang, G. Cai, Y. Qiu et al., "Structural segregation of gut microbiota between colorectal cancer patients and healthy volunteers," ISME Journal, vol. 6, no. 2, pp. 320-329, 2012. 
[9] I. Sobhani, J. Tap, F. Roudot-Thoraval et al., "Microbial dysbiosis in colorectal cancer (CRC) patients," PLoS ONE, vol. 6, no. 1, Article ID e16393, 2011.

[10] J. R. Marchesi, B. E. Dutilh, N. Hall et al., "Towards the human colorectal cancer microbiome," PLoS ONE, vol. 6, no. 5, Article ID e20447, 2011.

[11] J. J. Farrell, L. Zhang, H. Zhou et al., "Variations of oral microbiota are associated with pancreatic diseases including pancreatic cancer," Gut, vol. 61, no. 4, pp. 582-588, 2012.

[12] H. S. Said, W. Suda, S. Nakagome et al., "Dysbiosis of salivary microbiota in inflammatory bowel disease and its association with oral immunological biomarkers," DNA Research, vol. 21, no. 1, pp. 15-25, 2014.

[13] B. C.-Y. Wong, S. K. Lam, W. M. Wong et al., "Helicobacter pylori eradication to prevent gastric cancer in a high-risk region of China: a randomized controlled trial," Journal of the American Medical Association, vol. 291, no. 2, pp. 187-194, 2004.

[14] D. B. Polk and R. M. Peek Jr., "Helicobacter pylori: gastric cancer and beyond," Nature Reviews Cancer, vol. 10, no. 6, pp. 403-414, 2010.

[15] W.-Q. Li, J.-L. Ma, L. Zhang et al., "Effects of Helicobacter pylori treatment on gastric cancer incidence and mortality in subgroups," Journal of the National Cancer Institute, vol. 106, no. 7, Article ID dju116, 2014.

[16] M. Wang, Q. Zhang, J. Zhu, Y. Tao, Q. Kong, and H. Shang, "A new computerized tongue diagnosis method with optimized outline extraction algorithm using HSV color model," Journal of Computational and Theoretical Nanoscience, vol. 11, no. 6, pp. 1556-1562, 2014.

[17] Q. Wang, G. M. Garrity, J. M. Tiedje, and J. R. Cole, "Naïve Bayesian classifier for rapid assignment of rRNA sequences into the new bacterial taxonomy," Applied and Environmental Microbiology, vol. 73, no. 16, pp. 5261-5267, 2007.

[18] R. C. Edgar, "UPARSE: highly accurate OTU sequences from microbial amplicon reads," Nature Methods, vol. 10, no. 10, pp. 996-998, 2013.

[19] R. C. Edgar, B. J. Haas, J. C. Clemente, C. Quince, and R. Knight, "UCHIME improves sensitivity and speed of chimera detection," Bioinformatics, vol. 27, no. 16, pp. 2194-2200, 2011.

[20] C. Quast, E. Pruesse, P. Yilmaz et al., "The SILVA ribosomal RNA gene database project: improved data processing and webbased tools," Nucleic Acids Research, vol. 41, no. 1, pp. D590D596, 2013.

[21] R. E. Ley, F. Bäckhed, P. Turnbaugh, C. A. Lozupone, R. D. Knight, and J. I. Gordon, "Obesity alters gut microbial ecology," Proceedings of the National Academy of Sciences of the United States of America, vol. 102, no. 31, pp. 11070-11075, 2005.

[22] N. Larsen, F. K. Vogensen, F. W. J. van den Berg et al., "Gut microbiota in human adults with type 2 diabetes differs from non-diabetic adults," PLoS ONE, vol. 5, no. 2, Article ID e9085, 2010.

[23] J. Steevens, L. J. Schouten, R. A. Goldbohm, and P. A. van den Brandt, "Alcohol consumption, cigarette smoking and risk of subtypes of oesophageal and gastric cancer: a prospective cohort study," Gut, vol. 59, no. 1, pp. 39-48, 2010.

[24] I. Tramacere, E. Negri, C. Pelucchi et al., "A meta-analysis on alcohol drinking and gastric cancer risk," Annals of Oncology, vol. 23, no. 1, Article ID mdr135, pp. 28-36, 2012.

[25] V. Lazarevic, K. Whiteson, S. Huse et al., "Metagenomic study of the oral microbiota by Illumina high-throughput sequencing," Journal of Microbiological Methods, vol. 79, no. 3, pp. 266-271, 2009.
[26] C. E. Kazor, P. M. Mitchell, A. M. Lee et al., "Diversity of bacterial populations on the tongue dorsa of patients with halitosis and healthy patients," Journal of Clinical Microbiology, vol. 41, no. 2, pp. 558-563, 2003.

[27] F. E. Dewhirst, T. Chen, J. Izard et al., "The human oral microbiome," Journal of Bacteriology, vol. 192, no. 19, pp. 50025017, 2010.

[28] B. Signat, C. Roques, P. Poulet, and D. Duffaut, "Role of Fusobacterium nucleatum in periodontal health and disease," Current Issues in Molecular Biology, vol. 13, no. 2, pp. 25-36, 2011.

[29] B. L. Pihlstrom, B. S. Michalowicz, and N. W. Johnson, "Periodontal diseases," The Lancet, vol. 366, no. 9499, pp. 1809-1820, 2005.

[30] J. Ahn, R. Sinha, Z. Pei et al., "Human gut microbiome and risk for colorectal cancer," Journal of the National Cancer Institute, vol. 105, no. 24, pp. 1907-1911, 2013.

[31] P. B. Lockhart, M. T. Brennan, H. C. Sasser, P. C. Fox, B. J. Paster, and F. K. Bahrani-Mougeot, "Bacteremia associated with toothbrushing and dental extraction," Circulation, vol. 117, no. 24, pp. 3118-3125, 2008.

[32] C. C. Abnet, F. Kamangar, S. M. Dawsey et al., "Tooth loss is associated with increased risk of gastric non-cardia adenocarcinoma in a cohort of Finnish smokers," Scandinavian Journal of Gastroenterology, vol. 40, no. 6, pp. 681-687, 2005.

[33] M. Asqah, N. Hamoudi, and S. Anil, "Is the presence of Helicobacter pylori in the dental plaque of patients with chronic periodontitis a risk factor for gastric infection?" Canadian Journal of Gastroenterology, vol. 23, no. 3, pp. 177-179, 2009.

[34] J. Ahn, C. Y. Chen, and R. B. Hayes, "Oral microbiome and oral and gastrointestinal cancer risk," Cancer Causes and Control, vol. 23, no. 3, pp. 399-404, 2012.

[35] M. S. Meyer, K. Joshipura, E. Giovannucci, and D. S. Michaud, "A review of the relationship between tooth loss, periodontal disease, and cancer," Cancer Causes and Control, vol. 19, no. 9, pp. 895-907, 2008. 

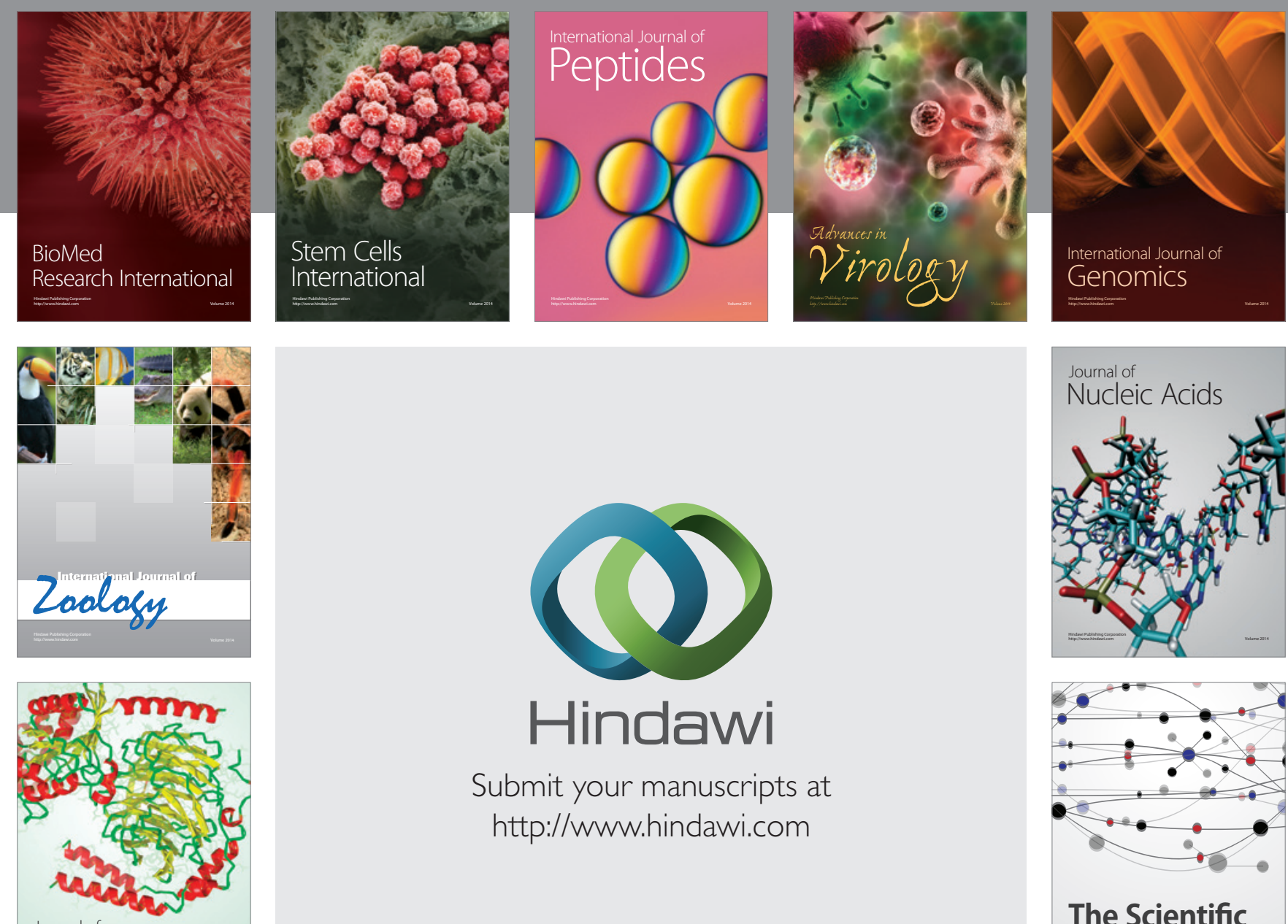

Submit your manuscripts at

http://www.hindawi.com

Journal of
Signal Transduction
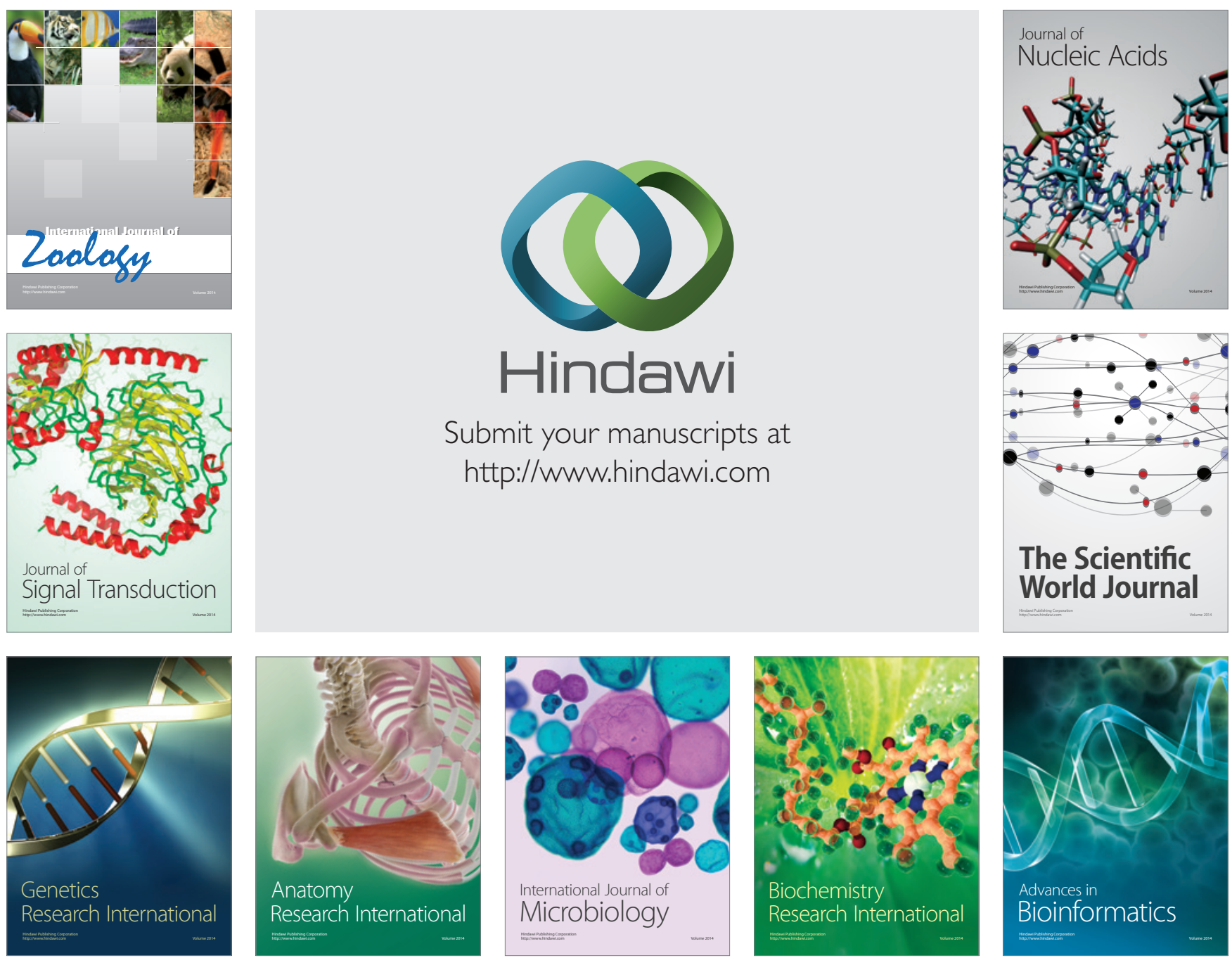

The Scientific World Journal
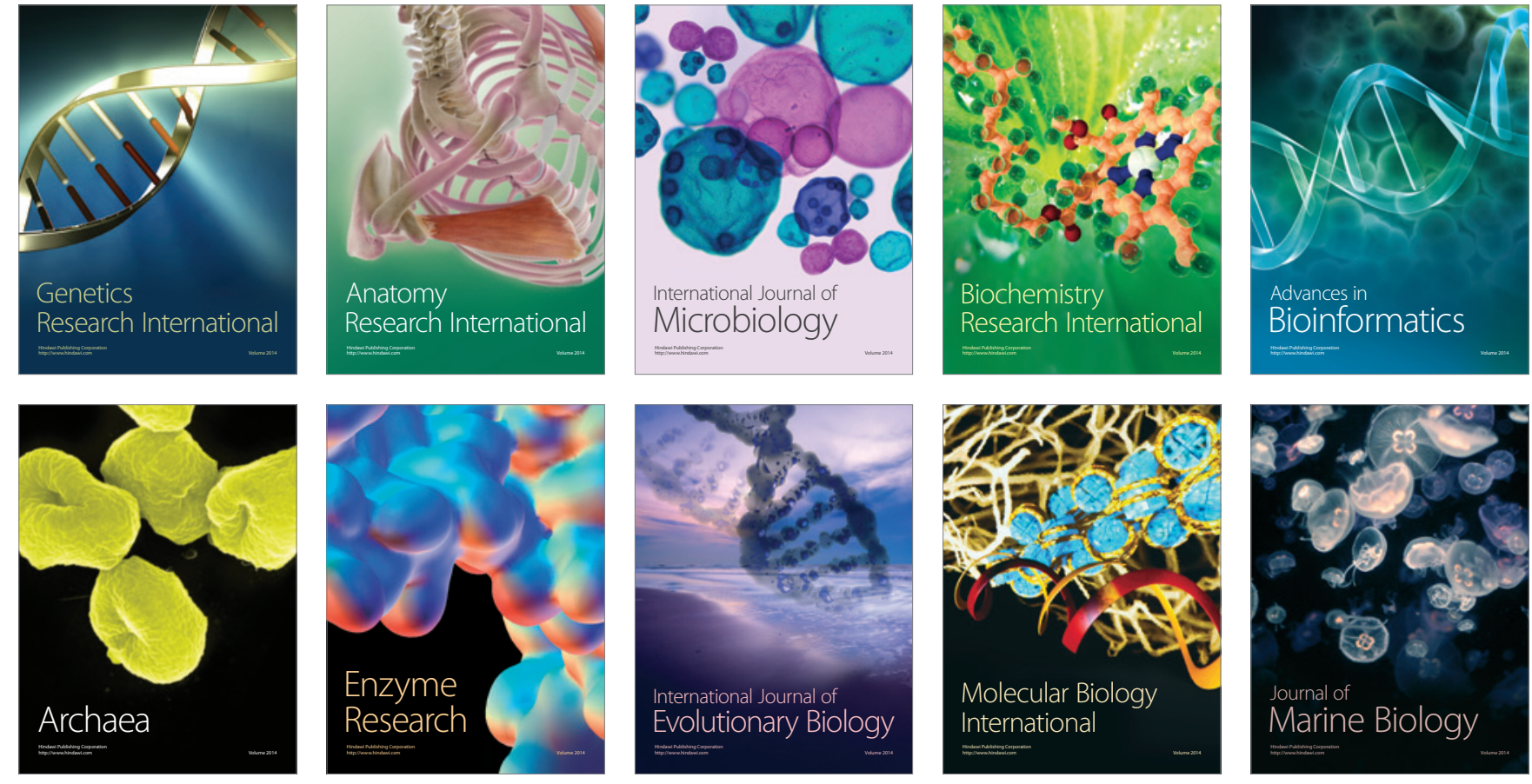